\title{
TERAPIA OCUPACIONAL EN ADICCIONES: DE LA TEORÍA A LA PRÁCTICA
}

\author{
OCCUPATIONAL THERAPY IN ADDICTION: FROM THEORY TO PRACTICE
}

\section{Gloria Rojo-Mota}

\section{Resumen}

La Terapia Ocupacional es una disciplina cuyo marco conceptual, objetivos y métodos están bien establecidos. Uno de los ámbitos en los que interviene es el de la adicción. Sin embargo, los textos y artículos ocupacionales disponibles carecen, en muchos casos, de un marco explicativo propio, recurriendo con demasiada frecuencia a otros importados de disciplinas preponderantes. Ello lleva, por una parte, a una pobre producción científica y, por otra, a un rol secundario de nuestra disciplina en los programas terapéuticos. El presente trabajo recoge la propuesta de un marco explicativo de la adicción desde una perspectiva ocupacional. Algunos autores parten de considerar que la propia adicción es, en sí misma, una ocupación, en la medida en que proporciona un rol y una significación al propio adicto. Desde esta perspectiva, toman sentido ocupacional paradigmas experimentales recientes, como el del enriquecimiento ambiental, o conceptos como el de empoderamiento. La rehabilitación cognitiva funcional, ampliamente aplicada en clínica del daño cerebral, representa otro enfoque complementario en la clínica de la adicción. Existen instrumentos de evaluación propios que cuantifican o sistematizan las variables ocupacionales relevantes. La Terapia Ocupacional está en condiciones de asumir un rol propio, protagonista e irrenunciable en el tratamiento de la adicción.

\section{Palabras clave:}

Terapia Ocupacional, Modelos Teóricos, Evaluación, Tratamiento, Adicción, Rehabilitación Funcional.

\begin{abstract}
Occupational Therapy is a discipline whose conceptual framework, objectives and methods are well established. Addiction is one of the areas in that is involved. However, the available occupational texts lacking in many cases its own explanatory framework, resorting too often to other imported from prevailing disciplines. This leads, on the one hand, to a poor scientific production and, on the other one, to a secondary role for our discipline in therapeutic programs. This paper presents a proposed explanatory framework of addiction from an occupational perspective. Some authors start considering that addiction is itself an occupation, to the extent that it provides a role and significance to the addict himself. From this perspective, it makes occupational sense some recent experimental paradigms, such as environmental enrichment, or concepts such as empowerment. The functional cognitive rehabilitation, widely applied in clinical of brain injury, represents another complementary approach to addiction clinic. There are own assessment instruments that measure or systematize the relevant occupational variables. Occupational Therapy is able to take its own role, main and indispensable prominence in the treatment of addiction.
\end{abstract}

\section{Keywords:}

Occupational Therapy, Theoretical Models, Assessment, Treatment, Addictive Behaviors, Functional Rehabilitation. 


\section{INTRODUCCIÓN}

Aun cuando la Terapia Ocupacional lleva décadas interviniendo en problemas relacionados con la adicción, su papel no siempre ha sido bien comprendido y suficientemente dignificado. La preeminencia de modelos asistenciales que consideran al adicto como "enfermo irrecuperable" ha llevado a esta disciplina a asumir roles secundarios, a recibir el encargo de "mantener ocupados" a los adictos, en lugar de utilizar la ocupación como medio y fin de su intervención. Sin embargo, el enfoque del adicto como persona que tiene dificultades en su relación con el entorno y que puede recuperarse y asumir roles adecuados, saludables y gratificantes, sitúa a la Terapia Ocupacional en primera línea de intervención (Rojo-Mota, 2008).

La Asociación Americana de Terapia Ocupacional define esta disciplina como el "conjunto de técnicas, métodos y actuaciones que, a través de actividades aplicadas con fines terapéuticos, previene y mantiene la salud, favorece la restauración de la función, suple las deficiencias incapacitantes y valora los supuestos del comportamiento y su significación profunda para conseguir las mayores independencia y reinserción posibles del individuo en todos sus aspectos: laboral, mental, físico y social" (AOTA, 2012). Por su parte, la Federación Mundial de Terapeutas Ocupacionales (WFOT), la define como la "profesión que se ocupa de la promoción de la Salud y el Bienestar a través de la ocupación. El principal objetivo de la terapia ocupacional es capacitar a las personas para participar en las actividades de la vida diaria. Los terapeutas ocupacionales logran este resultado mediante la habilitación de los individuos para realizar aquellas tareas que optimizarán su capacidad para participar, o mediante la modificación del entorno para que éste refuerce la participación" World Federation of Occupational Therapists, 2012). Finalmente, la Asociación Profesional Española de Terapia Ocupacional, la considera "una profesión socio-sanitaria que a través de la valoración de las capacidades y problemas físicos, psíquicos, sensoriales y sociales del individuo pretende, con un adecuado tratamiento, capacitarle para alcanzar el mayor grado de independencia posible en su vida diaria, contribuyendo a la recuperación de su enfermedad y/o facilitando la adaptación a su discapacidad" (APETO, s. f.).

La Terapia Ocupacional es una disciplina que nace con sus raíces en la Medicina y la Psicología. Sin em- bargo, la acumulación de conocimientos, técnicas y conceptos sobre la importancia de la ocupación humana y su relación con la salud, permite afirmar, en el momento actual que existe, más allá de los aspectos terapéuticos, lo que se ha denominado la Ciencia Ocupacional, un cuerpo de conocimiento y de investigación, que permite a los profesionales pensar y expresarse en términos ocupacionales, prescindiendo de la conceptualización ajena a la propia Terapia Ocupacional (Wilcock, 2001). El concepto central de esta ciencia es la consideración del ser humano como un ser ocupacional: la Ciencia Ocupacional es la ciencia de la vida cotidiana. Una disciplina académica, que se centra en los beneficios de la actividad productiva, social y física ("ocupaciones") en la vida cotidiana de las personas. Se dedica a aprender cómo la participación en la ocupación puede responder a las preocupaciones de salud global del Siglo XXI mediante la promoción de la salud, el bienestar y calidad de vida a lo largo de la vida. En definitiva, la Ciencia de la Ocupación consiste en el estudio sistemático de los humanos como seres ocupacionales, incluyendo la necesidad y la capacidad de adaptarse e involucrarse, y de estructurar sus ocupaciones para dar forma a sus vidas. Sus objetivos son por tanto, generar una base sistemática de descripción y comprensión de la participación de las personas en sus ocupaciones; y proveer el conocimiento básico que apoye la práctica aplicada de la Terapia Ocupacional (Navarrete Salas, 2010).

Uno de los campos de interés en esta novedosa Ciencia de la Ocupación es el estudio de los hábitos como elementos aprendidos y automatizados de la ocupación humana, que adquieren sentido en un contexto ecocultural determinado, con el que se involucran en una relación dialéctica, y se relacionan con el significado personal, la identidad, la competencia, la satisfacción y la auto-expresión; la Ciencia Ocupacional investiga y sintetiza el conocimiento interdisciplinario sobre los hábitos, incluyendo la identificación de las condiciones ambientales que mejoran la ocupación, el potencial ambiental, la contribución de los hábitos a la salud o a su deterioro, la identificación de las fortalezas, capacidades e intereses personales y su recuperación cuando existe un deterioro y la reconstrucción del yo a través de cambio de hábitos (Yerxa, 2002). 


\section{La adicción}

La adicción es un fenómeno comportamental complejo y multideterminado, que implica elementos genéticos, epigenéticos, del desarrollo, educacionales, psicológicos, ambientales y culturales, que se caracteriza por el establecimiento de hábitos o conductas repetitivas que escapan a los mecanismos de supervisión cerebral de la conducta, de modo que se repite por sus efectos reforzantes a corto plazo, aun cuando acarree malas consecuencias posteriores. En términos neuropsicológicos, la adicción se explica, en parte, por la alteración en el funcionamiento de diversas estructuras cerebrales, especialmente una disminución de la actividad de la corteza prefrontal en la regulación de los inputs procedentes de niveles cerebrales inferiores (Volkow y Baler, 2014). Diversos modelos neuropsicológicos de la adicción enfatizan el papel de la corteza prefrontal, cuyo "apagado" favorece la pérdida de control sobre la conducta, la automatización de los comportamientos asociados al reforzador (Redish, Jensen y Johnson, 2008; Goldstein y Volkow, 2002) y, en último término, estableciendo hábitos mediante los circuitos cerebrales implicados (Newlin y Strubler, 2007). Este déficit funcional de la corteza prefrontal puede ser previo, como consecuencia de un historial de aprendizaje deficitario, operando entonces como mecanismo favorecedor de la adicción (Olivar Arroyo, Ruiz Sánchez de León y Pedrero Pérez, 2014), o consecuencia del propio proceso adictivo (Chambers, Taylor y Potenza, 2003), incluyendo los efectos del estrés (Arnsten, 2009), siendo lo más probable la combinación de ambos (Ersche, Williams, Robbins y Bullmore, 2013). Este "apagado" o déficit funcional prefrontal, se manifiesta en diversas alteraciones en procesos vinculados a esta estructura, como son la atención, la inhibición de conductas inapropiadas, la planificación de la conducta, el seguimiento de planes, la estimación de consecuencias o la supervisión general de la conducta (Goldstein y Volkow, 2011), frecuentemente asociados a la adicción (Rojo-Mota, Pedrero-Pérez, Ruiz-Sánchez de León, Llanero-Luque y Puerta-García, 2013), independientemente de que implique el uso de sustancias o la mera repetición de conductas, como el juego o la adicción al sexo (Kelley y Berridge, 2002).

Durante décadas ha prevalecido la consideración de que la adicción es una enfermedad mental/cerebral, de carácter crónico y de curso recidivante, basada en predisposiciones genéticas y ligada a otros trastornos mentales (Leshner, 1997). Sin embargo, en el momento actual esa consideración ha sido fuertemente criticada y todos sus principios falsados, existiendo un acuerdo en que las alteraciones neurológicas y neurocomportamentales son sólo un aspecto de la adicción, que debe entenderse como una manera, más o menos acertada, de afrontar las dificultades de la vida, y que, en todo caso, refleja "un trastorno de la persona en su relación con un entorno determinado": un trastorno recuperable, una persona que puede manejar de modo más adecuadas sus dificultades y un entorno que puede ser modificado para favorecer la plena integración del individuo (Hall, Carter y Forlini, 2015; Levy, 2013; Satel y Lilienfeld, 2014).

La adicción, en el momento actual, se entiende como un hábito, una conducta aprendida y automatizada, que en su inicio tenía características adaptativas, pero, mediante la generalización a situaciones en las que su aplicación es inadecuada genera consecuencias indeseadas (Newlin y Strubler, 2007). Están bien identificadas las vías neuronales que intervienen en la transición entre la conducta, el hábito y la compulsión (Everitt y Robbins, 2005). Sin embargo, hay pocos estudios que exploren la relación entre el consumo de sustancias y la afectación ocupacional que genera su abuso o dependencia.

\section{Terapia Ocupacional y Adicción}

Algunos estudios han explorado la conducta adictiva como una ocupación en sí misma, de modo que la búsqueda de financiación para la obtención de las dosis, su adquisición, su autoadministración y la obtención de sus efectos configuran, de modo exclusivo, la ocupación de la vida diaria del adicto, compitiendo con otras ocupaciones que quedan relegadas o abandonadas (Sackman, Sackman y DeAngelis, 1978). De entre las múltiples definiciones que se han propuesto para el término ocupación, algunas se aproximan significativamente al propio concepto de adicción: la ocupación como actividad autoiniciada, autoorganizada y dirigida a metas contextualizadas en un ambiente específico que se desarrollan en periodos de tiempo muy prolongados (Yerxa, 2000). La ocupación también se entiende como "un conjunto de actividades que se realiza con consistencia y regularidad; que presenta una estructura y un valor y significado para los individuos en un contexto cultural" (Polatajko et al., 2007). Es obvio que no todas las ocupaciones son necesariamente beneficiosas, y que algunas, si bien pueden aportar valor y sentido a la vida de un individuo en particular, son mal valorados 
por la cultura, y no contribuyen a la salud y el bienestar del individuo (Polatajko et al., 2007). Sin embargo, para los adictos, el consumo de drogas es una ocupación en la medida en que pueden desarrollar una percepción de control, el alivio temporal del malestar emocional y físico, la aceptación y la interacción entre iguales, factores que pueden ser considerados como un propósito (Herie, Godden, Shenfeld y Kelly, 2007). Considerado como tal, la adicción es objeto de intervención para la Terapia Ocupacional (Kiepek y Magalhães, 2011).

Cuando una disciplina pretende abordar un problema, necesita esquematizar su comprensión sobre la problemática objeto de intervención en un modelo comprehensivo que facilite el diseño del trabajo a desa- rrollar. Con frecuencia, la Terapia Ocupacional recurre a modelos ajenos para la comprensión del problema, aplicando después técnicas propias para la intervención. Esto supone, en último término, que la disciplina "interviene sobre cuestiones que no comprende", algo inadmisible cuando pretende enmarcarse en una ciencia propia, la Ciencia Ocupacional.

Helbig y McKay (2003) han propuesto un modelo teórico desde la Terapia Ocupacional para la explicación del comportamiento adictivo (Figura 1). Previamente Wilcock (1998) identificó tres amenazas para el mantenimiento de la salud: el desequilibrio ocupacional, la deprivación ocupacional y la alienación ocupacional.

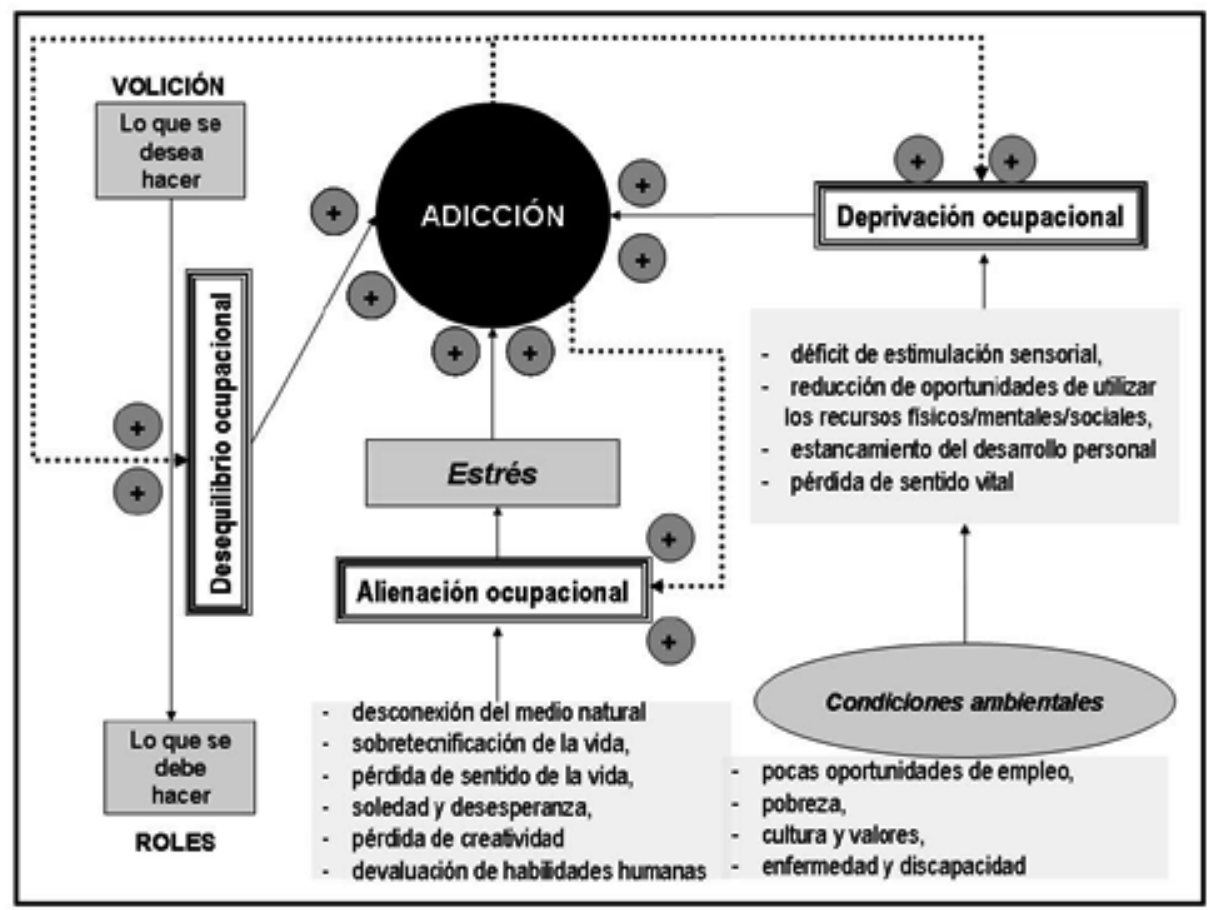

Figura 1. Esquema del modelo ocupacional de la adicción de Helbig y McKay [27].

El desequilibrio ocupacional sucede cuando los individuos son incapaces de ajustar sus necesidades físicas, sociales y mentales a sus roles asumidos. El desajuste entre "lo que se debe hacer" y "lo que se desea hacer" puede llevar a la persona a optar por las opciones vinculadas a la adicción, de modo que el "rol de adicto" adquiere un protagonismo tal que impide la asunción de roles alternativos o complementarios. Muchos individuos pueden experimentar este equilibrio debido a las condiciones sociales, ocupacionales o ambientales, que no proporcionan el mismo valor o significado a las diferentes opciones, de modo que el estrecho margen de ocupaciones en la vida diaria que acompañan a la adicción puede favorecer el que el in- 
dividuo opte por la simplificación y justifique el consumo como ocupación, incorporándose o reintegrándose al ciclo adictivo. Paradójicamente, esta adicción-comoocupación puede proporcionar al adicto la fuente principal de identidad y sentido en la vida diaria, de modo que el "rol de adicto" configure su propia identidad.

La segunda amenaza es la deprivación ocupacional, que ocurre como resultado de fuerzas externas que dificultan la participación en ocupaciones saludables o normalizadas. Entre estas fuerzas externas al individuo están la pobreza, la falta de oportunidades educativas o laborales, los valores propios de cada cultura, las exigencias del mercado, etc. Esta deprivación no es producto de un momento determinado, sino que se va estableciendo lenta y progresivamente como consecuencia del empobrecimiento ambiental y la persistente falta de oportunidades y alternativas, resultando en carencias cognitivas, disminución de las capacidades físicas, intelectuales y sociales, dificultando el desarroIlo personal y deteriorando el propio sentido del sí mismo y de la vida en general.

La tercera amenaza es la alienación ocupacional, provocada por una excesiva tecnificación de la vida, que aísla al sujeto de su realidad ambiental en la medida en que dificulta su comprensión sobre los mecanismos de funcionamiento de la vida cotidiana, devalúa sus propias capacidades y recursos, empuja a un mundo competitivo en los que quienes han tenido menos oportunidades son excluidos con más facilidad, y provocan un estrés que el individuo debe afrontar de alguna manera. El uso de drogas, en este caso, se configura como un mecanismo de afrontamiento útil en el corto plazo, sin que el individuo haya aprendido o sea capaz de rentabilizar modalidades de afrontamiento alternativas con mejores resultados en el largo plazo. La reducción inmediata del estrés, mediante el escape o la evitación del malestar, refuerza el uso de drogas en la medida en que permite manejar la realidad en lo cotidiano.

Este modelo tiene dos implicaciones principales: por una parte, es un planteamiento realizado desde la conceptualización propia de la Terapia Ocupacional; por otra, aleja la adicción del territorio de la "enfermedad mental o cerebral", situando su etiología en factores ambientales y en la interacción que los individuos realizan permanentemente con éstos. De este modo, el modelo se vincula a paradigmas experimentales muy recientes, que consideran que la adicción es producto de la pobreza ambiental, de modo que su abordaje debe centrarse en tareas que produzcan un enriquecimiento ambiental: hay suficiente evidencia científica para considerar que el enriquecimiento ambiental tiene efectos superiores a otras intervenciones en la prevención y el tratamiento de las conductas adictivas (Solinas, Thiriet, Chauvet y Jaber, 2010). Ello permite desvincular a la Terapia Ocupacional de planteamientos obsoletos, basados en modelos de enfermedad psiquiátrica, que obligan a adoptar una terminología ajena a la Ciencia Ocupacional, como las propuestas de Gutman (2006).

En definitiva, la Terapia Ocupacional busca que el sujeto con conductas adictivas mejore su funcionamiento global en sus ocupaciones en la vida diaria, consiguiendo de este modo la independencia funcional, concepto que engloba, por una parte, factores personales (competencia, control, seguridad), por otra, elementos ambientales (contexto, cultura) y, en tercer lugar, componentes del continuo persona-ambiente (Bonikowsky, Musto, Suteu, MacKenzie y Dennis, 2012).

\section{MODELO DE INTERVENCIÓN DE LA TERAPIA}

\section{OCUPACIONAL EN LA ADICCIÓN}

La intervención de Terapia Ocupacional en el tratamiento de personas con conductas adictivas debe desglosarse en dos aspectos principales: la evaluación y el tratamiento.

\section{1) Evaluación}

La evaluación es el proceso mediante el cual se ponen en evidencia los aspectos ocupacionales que están siendo afectados por la conducta adictiva, los factores que pudieron determinar la instauración de ésta, las capacidades que han permanecido íntegras y que pueden ser el punto de apoyo para la recuperación, y las metas que es razonable proponer para el proceso de rehabilitación. Esta evaluación debe ser holística e individualizada, intentando captar el mayor número posible de factores que intervengan en el proceso adictivo, en su instauración, en su mantenimiento y en su recuperación. Al inicio del tratamiento debe suponer una valoración transversal, retrospectiva e idiográfica , pero debe también ser el punto de partida de una evaluación longitudinal que permita, según avanza el tratamiento, medir los cambios que se vayan produciendo, de modo que pueda cuantificarse el impacto de la intervención sobre los aspectos críticos sobre los que va 
incidiendo cada componente del programa de rehabilitación. Además, los instrumentos deben tener en cuenta tanto elementos observables (p. ej., rendimiento en tareas) como subjetivos, a partir de la experiencia del propio sujeto, en busca de una coherencia entre la mejoría aparente y la vivencia individual de recuperación.

Uno de los grandes problemas padecidos por la Terapia Ocupacional en su aplicación a los trastornos adictivos ha sido la ausencia casi total de instrumentos de evaluación (Rojo Mota, 2013a), lo que no permite cuantificar ni los problemas sobre los que intervenir, ni los efectos de la intervención. Y ello a pesar de la variedad de técnicas e instrumentos que la disciplina ha utilizado y validado en muchos otros ámbitos de trabajo (Reed, 2002).

Siguiendo lo ya expuesto (Bonikowsky et al., 2012), la evaluación debe dirigirse a tres aspectos esenciales: los factores personales, los elementos ambientales y los componentes del continuo persona-ambiente. Entre los instrumentos que pueden utilizarse destacan los siguientes: a) Rendimiento cognitivo. Los más recientes modelos neuropsicológicos de la adicción sitúan como antecedente directo de la pérdida de control que caracteriza a la adicción, el fallo en los sistemas prefrontales de control superior de la conducta (Figura 2; Yücel et al., 2008). Estos fallos se traducen en fallos de la planificación de la actividad cotidiana, tendencia a la irreflexividad, falta de iniciativa y motivación, pobre conciencia de problema, conducta desorganizada y no auto-supervisada, dificultades en el mantenimiento de la atención, dificultades en el proceso de abstracción, déficits en el comportamiento empático, dificultad para comprender instrucciones, incapacidad para generar estrategias alternativas, etc. Como el propio modelo sugiere, estos fallos son previos al establecimiento de la adicción (vulnerabilidades), debidas a procesos de aprendizaje deficitario o a cuestiones biológicas, pero se ven agravados por el propio proceso adictivo.

Figura 2.

Modelo neuropsicológico de la adicción [34]

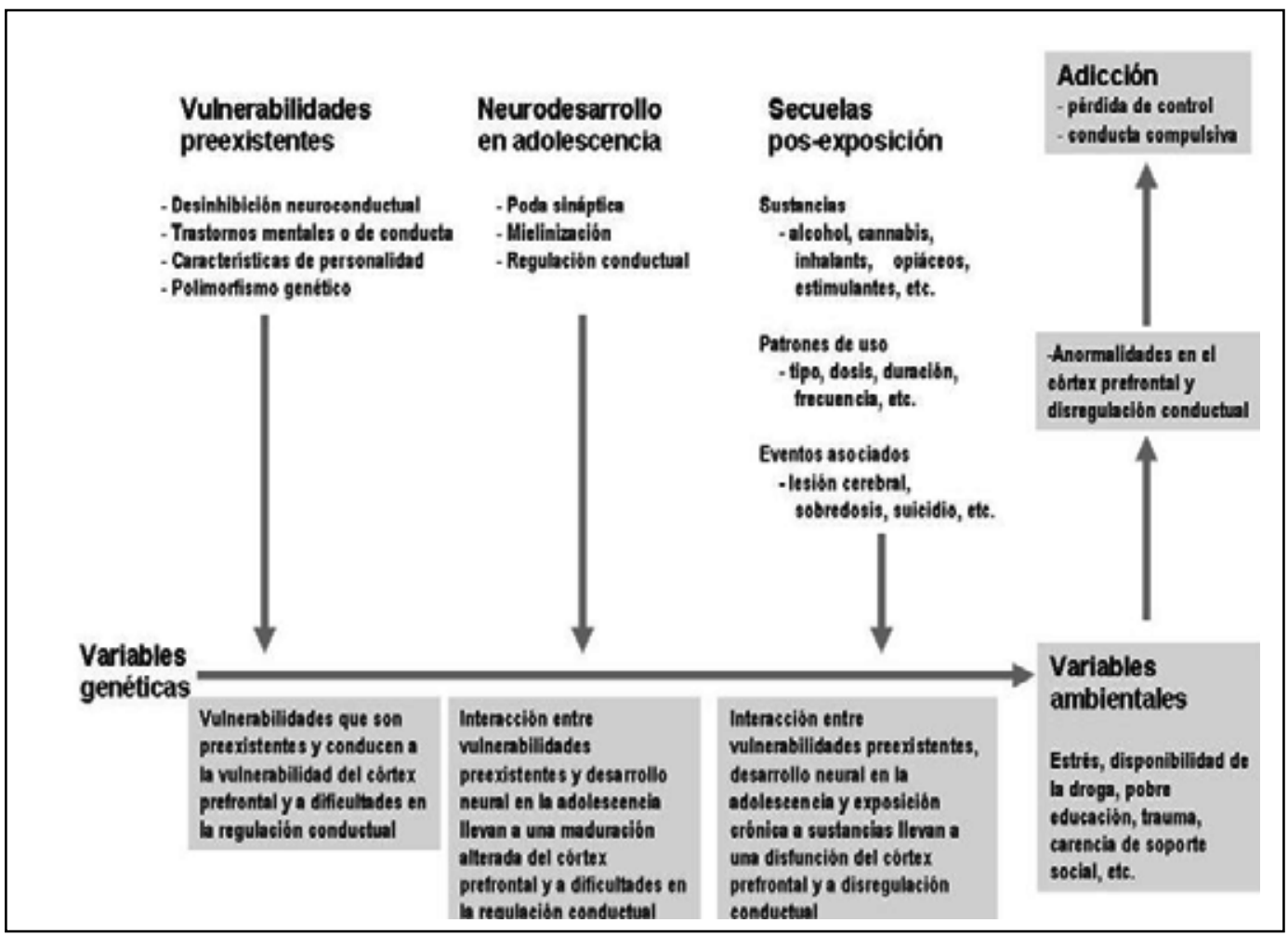


La evaluación de estos aspectos en profundidad no es competencia directa de la Terapia Ocupacional, pero se requiere un conocimiento de los déficits, así como de las funciones que permanezcan íntegras y sirvan de apoyo a las intervenciones encaminadas a la recuperación. Para la evaluación pueden utilizarse pruebas generales de cribado (screening), que nos informen de si el rendimiento del sujeto responde a lo esperable para sus condiciones socioculturales o muestra algún grado de déficit; entre estas pruebas se encuentra el Montreal Cognitive Assessment (MoCA; Nasreddine et al., 2005), la prueba más utilizada actualmente y que ha desplazado al tradicional MINIMENTAL (Carnero-Pardo, 2014) y se ha utilizado con éxito en población española con conductas adictivas (Rojo-Mota et al., 2013). No obstante, si se requiere una evaluación más en profundidad del funcionamiento cognitivo del paciente con conducta adictiva, la Terapia Ocupacional dispone de instrumentos propios para desarrollarla, como la LOTCA o la COTNAB entre otras (Sánchez Durán, 2011).

La detección temprana de estos déficits cognitivos es crítica, en la medida en que muchos abandonos de tratamiento se producen porque se pide a la persona que lleve a cabo tareas que simplemente no se encuentra en condiciones de hacer, lo que provoca frustración y pérdida de motivación para el cambio. Cuantificar y localizar los déficits permite intervenir, de forma graduada y progresiva, para situar al sujeto en mejores condiciones de aprovecharse del resto de componentes del programa de recuperación (Aharonovich et al., 2006).

b) Historia ocupacional. Se trata de recoger todos los elementos que han ido configurando la identidad ocupacional del sujeto, su percepción de competencia y la influencia de los diferentes ambientes en su desarrollo ocupacional. Es habitual encontrar que el establecimiento de una conducta adictiva supone la renuncia a ocupaciones que habían resultado significativas y gratificantes en el pasado, otras que han aparecido asociadas al consumo y han ocupado su lugar o las han desplazado por competencia temporal. En este aspecto se sugiere el uso de la Entrevista Histórica de Desempeño Ocupacional (OPHI-II; Kielhofner et al., 1998).

c) Desempeño ocupacional actual. El objetivo es evaluar el grado de calidad en el desempeño actual, desde la perspectiva del propio sujeto. Se trata de establecer una línea base a partir de la cual establecer las prioridades del tratamiento, la graduación de la exigencia en el desempeño, la propia percepción del sujeto. El Autoinforme del Desempeño Ocupacional (ADO; Rojo-Mota, Pedrero-Pérez, Ruiz-Sánchez de León, Lla-
nero-Luque y Puerta-García, 2011) es una herramienta compuesta por dos partes con dos estimaciones cada una. La primera parte es de autoevaluación ocupacional en la que los participantes estiman su desempeño ocupacional y posteriormente valoran este desempeño. A continuación, se procede a una evaluación del ambiente y su desempeño en tales condiciones. La herramienta se divide en siete subescalas diferenciada por ítems (Planificación, Autonomía, Físico relacional, Flexibilidad, Necesidades básicas, Recursos y Entorno). El cuestionario permite aislar tanto los aspectos deficitarios como las fortalezas percibidas por los propios sujetos. El cuestionario se ha utilizado ya en trabajos con sujetos con adicciones (de León Rodríguez, Cantero Garlito y Zabala Baños, 2013).

Si el autoinforme nos proporciona una visión subjetiva, desde la consideración del propio sujeto, disponemos también de otros instrumentos que nos permiten objetivar la calidad de ejecución en tareas de la vida diaria. El AMPS (Assessment of Motor and Process Skills, Fisher, 1995) es la prueba considerada como el gold standard de la evaluación sobre la calidad de ejecución en tareas cotidianas. A pesar de que requiere de una in tensa formación previa para garantizar la correcta administración, y de ciertas condiciones para su aplicación en la clínica, existen estudios que muestran su aplicabilidad en personas tratadas por adicción (Rojo-Mota, Pedrero-Pérez, Ruiz-Sánchez de León y Miangolarra Page, 2014). Otras pruebas pueden permitirnos clasificar el rendimiento de los sujetos, su capacidad de aprendizaje y trazar el recorrido hasta la independencia funcional; tal es el caso del ACLS-5 (Allen Cognitive Level Screen-5 (Allen et al., 2007), también aplicado con éxito en personas tratadas por adicción (Rojo-Mota, Pedrero-Pérez, Huertas-Hoyas, Merritt y MacKenzie, 2015).

\section{2) Tratamiento}

La evaluación nos proporciona el punto de partida, el estado actual del sujeto, las metas a conseguir y la graduación de las intervenciones a desarrollar. Un error muy común en la clínica es considerar que "todos los adictos tienen problemas comunes" y graduar la intervención en función del momento terapéutico (desintoxicación, deshabituación y reinserción) y/o de la droga consumida (grupos de heroína, cocaína y alcohol). La experiencia clínica nos muestra como cada persona presenta un ritmo propio de recuperación, con independencia de la sustancia consumida o de las conduc- 
tas problemáticas (adicciones sin sustancia). Por tanto, es preciso partir de la evaluación inicial para diseñar una intervención individualizada, que debe cumplir una serie de requisitos: (a) Las experiencias que proporcione el tratamiento, para que produzcan una marcación positiva, deben contar con significación, tener valor para la persona; (b) deben tener vinculación con su realidad diaria; y (c) deben ser graduadas, relacionando dificultad y capacidades, de forma que se garantice el éxito en su ejecución.

Los objetivos globales de la intervención son (Figura 3): (a) rehabilitación cognitiva funcional, (b) el empoderamiento del paciente, y (c) el enriquecimiento ambiental.

Figura 3.

Objetivos globales de la intervención de Terapia Ocupacional en adicciones

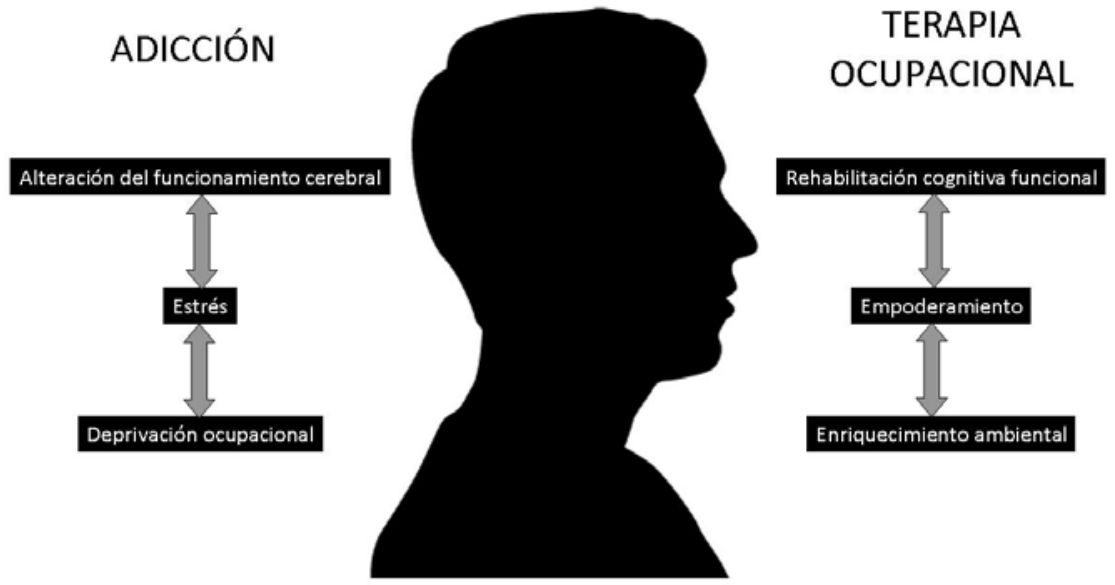

a) Rehabilitación cognitiva. La rehabilitación cognitiva se configura como una técnica prometedora en los programas de tratamiento de personas adictas. Así lo indican los aún pocos trabajos publicados sobre resultados de su aplicación. Los efectos parecen operar mediante dos vías: una directa, favoreciendo el control superior de la conducta $y$, en consecuencia, incrementando el control de comportamientos automatizados (entre ellos, los vinculados a la adicción) y reduciendo las consecuencias negativas de tales automatismos (lo que incluye conductas psicopatológicas, como la depresión, entre otras); y una vía indirecta, favoreciendo el mejor aprovechamiento del resto de actividades del plan terapéutico, en especial las técnicas cognitivo-conductuales de prevención de recaídas. Se han propuesto dos modalidades de intervención: una, más focalizada en las funciones cerebrales alteradas al hilo de la adicción (tanto las que favorecieron su instauración como las implicadas en su mantenimiento) y otra, más comprometida con el funcionamiento en tareas reales de la vida cotidiana, en dónde se traducen los déficits funcionales ya descritos. Esta segunda es la intervención propia de la Te- rapia Ocupacional: la rehabilitación cognitiva funcional. Ambas cuentan con programas de aplicación, si bien hay pocos trabajos que hayan explorado su eficacia en sujetos adictos, ambas requieren un alto grado de individualización y ambas son complementarias y no excluyentes, siendo preciso contar con trabajos que estudien qué tipo de sujetos pueden beneficiarse más de una u otra o de la combinación de ambas. Estas técnicas pueden incorporarse a programas biopsicosociales y multidisciplinares, y de hecho sólo tienen sentido cuando forman parte de este tipo de intervenciones que tienen en cuenta la globalidad del sujeto incorporado a su ambiente. Los programas computarizados favorecen la investigación de resultados, mientras que los programas funcionales aportan validez ecológica en la medida en que tienen en cuenta las relaciones establecidas entre cada sujeto y su ambiente particular (Pedrero-Pérez, Rojo-Mota, Ruiz-Sánchez de León, Llanero-Luque y Puerta-García, 2011; Rojo Mota, Iraurgi Castillo y Sánchez Cabeza, 2011). Los principios básicos que deben regir la aplicación de estos programas son: (a) el paciente debe ser el protagonista de la intervención; (b) la 
intervención debe ir dirigida al contexto; (c) la intervención debe estar basada en la ocupación; (d) toda intervención debe estar basada en la evidencia científica, ya sea disponible o con intención de generarla; (e) la evaluación debe ser dinámica y continua; $(\mathrm{f})$ debe iniciarse en fases tempranas de la intervención rehabilitadora; (g) se debe evitar el estrés del paciente, proporcionando desafíos graduados en dificultad y proporcionar una retroalimentación inmediata de los resultados; (h) debe buscarse una continuidad de las tareas, tanto en su desarrollo en el encuadre terapéutico como en su vinculación a las actividades de la vida diaria. El desarrollo de las sesiones, ya sean individuales o grupales, debe responder a una organización temporal, desde actividades preparatorias (p. ej., ejercicio físico o relajación), habilitadoras (entrenan las funciones que se pondrán en marcha en las fases posteriores), propositivas (desarrolladas en contexto terapéutico dirigidas a entrenar el desempeño cotidiano), hasta las verdaderas ocupaciones (tareas a desarrollar en la vida diaria entre sesión y sesión) (Pedretti, 1996).

b) Empoderamiento. El concepto de empoderamiento ha ido ganando terreno en múltiples ámbitos relacionados con la salud. Su definición general apunta a que se trata del proceso por el cual las personas fortalecen sus capacidades, confianza, visión y protagonismo como grupo social para impulsar cambios positivos de las situaciones que viven (HEGOA, 2006). En su aplicación en Ciencias de la Salud, el concepto presenta tres claras dimensiones: a) la personal, como desarrollo del sentido del sí-mismo, de la propia identidad, de la confianza y de la capacidad individual; b) la de las relaciones próximas, como capacidad de negociar e influir en la naturaleza de las relaciones y las decisiones, y c) la colectiva, como participación en la vida social y las estructuras comunitarias. Mediante este proceso, las personas perciben una relación más estrecha entre sus metas y el modo de alcanzarlas y una correspondencia entre sus esfuerzos y los resultados que obtienen (World Health Organization, 1998). El concepto de empoderamiento se opone a la sumisión y pérdida de identidad, credibilidad y expectativas de futuro que imponen las etiquetas psiquiátricas y las imágenes sociales asociadas a la locura o los problemas de salud mental. Por el contrario, este concepto se relaciona con los Modelos Orientados a la Recuperación, entendida ésta como un proceso de cambio a través del cual las personas mejoran su salud y bienestar, viven una vida auto-dirigida, y se esfuerzan para alcanzar su pleno potencial (World Health Organization, 1998). El Substance Abuse \& Mental Health Services Administration's (SAMHSA, 2004) definió las características que deben cumplir los programas orientados a la recuperación (Figura 4). Existen ya trabajos que aplican estos principios en el tratamiento de las adicciones (Hunter, Jason y Keys, 2013) y también modelos de intervención desde Terapia Ocupacional, aún no aplicados en este ám-

bito (Black y Wells, 2007).

Figura 4

Diez componentes fundamentales de los programas orientados a la recuperación (SAMHSA, 2004).

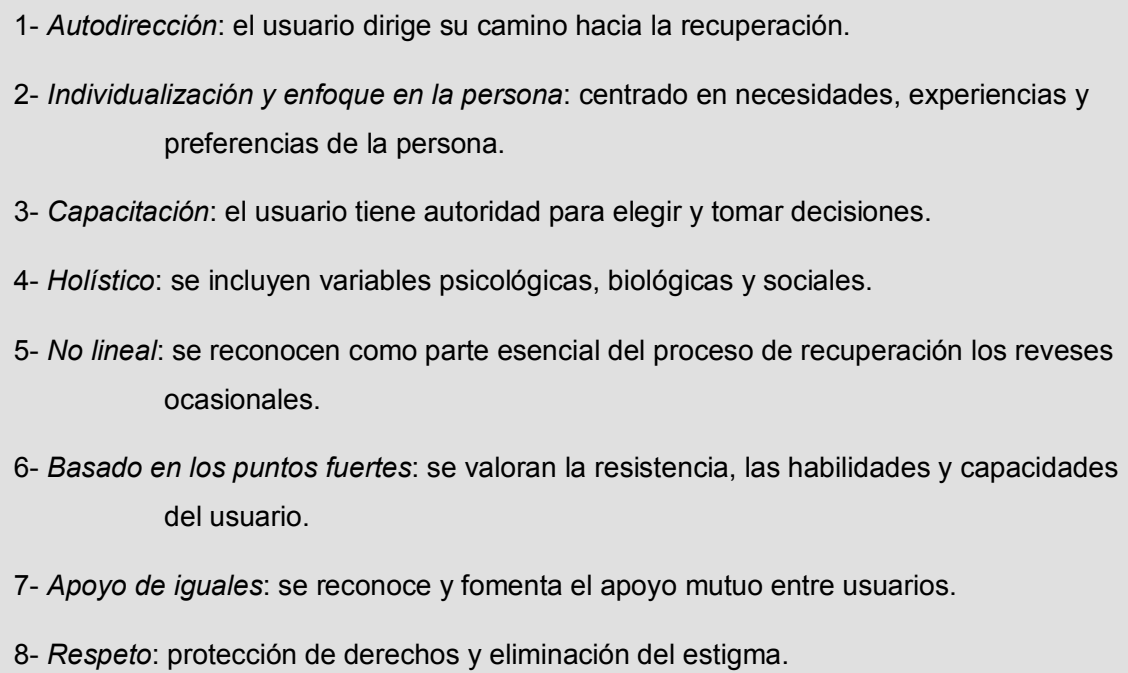


c) Enriquecimiento ambiental. La aparente vulnerabilidad a desarrollar una adicción resulta, en su mayor parte, de la ausencia de incentivos positivos (p. ej., recompensas alternativas al uso de drogas) y negativos (p. ej., costes) capaces de competir con la búsqueda de drogas. Ya en los años 70, Alexander propuso su estudio sobre el Rat Park: en el mostraba que animales que habían sido convertidos en adictos a la morfina en una caja de Skinner, dejaban espontáneamente de serlo cuando se les situaba en un ambiente en el que la droga seguía estando disponible, pero competía con muchos otros estímulos: juegos, comida, otros congéneres y la posibilidad de tener contactos sexuales, entre otros. Su trabajo encontró serías dificultades para ser publicado en las principales revistas científicas, consiguiéndolo, finalmente, en una de segunda fila (Alexander, Coambs y Hadaway, 1978). La propuesta era clara: la causa de la adicción no era la droga: era la caja. Durante décadas, su estudio permaneció silenciado dado que cuestionaba todas las creencias sobre los efectos de las drogas y sus posibles soluciones. De repente, toda la construcción en torno a los factores biológicos, genéticos, los neurotransmisores y las alteraciones cerebrales que supuestamente subyacían a la adicción, giró la vista hacia los factores ambientales, la epigenética y la adaptación a las dificultades de la vida. En la década pasada, se replicó su estudio bajo el denominado paradigma del enriquecimiento ambiental (Solinas, Thiriet, El Rawas, Lardeux y Jaber, 2009). La conclusión era clara: la adicción se esfumaba cuando el animal podía elegir entre diversos estímulos. Y ello resultó evidente no sólo para los opiáceos, como encontró Alexander, sino para cualquier droga conocida. Disponemos ya de propuestas para aplicar las estrategias de enriquecimiento ambiental en programas de prevención y tratamiento de las adicciones en humanos (Solinas et al., 2010). No disponemos aún de trabajos que evalúen el impacto del enriquecimiento ambiental en programas de Terapia Ocupacional, aunque sí interviniendo sobre el daño cerebral (Rao, 2014).

\section{CONCLUSIONES}

La Terapia Ocupacional lleva ya décadas interviniendo en problemas relacionados con la adicción. Sin embargo, la producción científica generada es muy escasa aún. La raíz de este problema puede encontrarse en la carencia de modelos propios para explicar el fe- nómeno sobre el que se interviene, lo que lleva a asumir modelos ajenos a la disciplina, principalmente del ámbito psiquiátrico. Los textos ocupacionales tienden a ignorar el cuerpo de conocimientos propios para "entregarse" a explicaciones importadas, que carecen de entidad ocupacional, sin que pueda atisbarse una conceptualización desde nuestra disciplina de los fenómenos sobre los que se interviene. En definitiva, se trata de evitar la comisión de un intrusismo que no aceptamos cuando se dirige hacia nuestra profesión.

Las teorías, y los modelos que las representan, explican los fenómenos describiendo la realidad subyacente y prediciendo nuevos fenómenos. La ausencia de teorías propias sitúa al terapeuta en el plano de la mera observación, la descripción de los fenómenos y la aplicación de la "experiencia clínica" individual, como si ello fuera suficiente para proporcionar una atención de calidad. La observación es un método imprescindible de trabajo, que sólo tiene utilidad en la medida en que confirma hipótesis previas (método hipotético deductivo) o genera conocimiento novedoso y contrastable a partir de la observación (método inductivo). La mera descripción de fenómenos, sin un método científico que permita relacionar las variables observadas, no genera conocimiento científico. Y la experiencia clínica individual, sin referencia a marcos teóricos comúnmente aceptados, está sujeta a sesgos y prejuicios difícilmente controlables. Una disciplina que renuncia al método científico y se entrega a la mera experiencia clínica está llamada a fracasar, o, como ha sucedido en algunos ámbitos para la Terapia Ocupacional, a no merecer el respeto y la consideración necesaria por parte de disciplinas preeminentes.

En este trabajo, se ha presentado un modelo explicativo de la adicción desde conceptos y premisas propias de la Terapia Ocupacional. Se ha mostrado también que nuestra disciplina cuenta con instrumentos propios que permiten cuantificar las variables observadas en términos estrictamente ocupacionales, tanto de la adicción como de los problemas de salud mental asociados (Rojo-Mota, 2013b), y también en problemas neurocomportamentales concretos (Rojo-Mota et al., 2009). De este modo, en lugar de recurrir a conceptos como tolerancia, dependencia, enfermedad o conducta, podemos explicar la adicción como ocupación, en términos de roles, voliciones, actividades significativas, calidad del desempeño, ambiente, etc.

A partir de esta evaluación no todo vale. La selección de actividades, la graduación de la dificultad y la generalización al ambiente real del sujeto deben res- 
ponder a las necesidades identificadas. El sujeto debe saber en todo momento cuál es el objetivo de lo que se está trabajando y sentirse en un proceso de mejora que debe traducirse en un incremento de su calidad de vida (de León et al., 2013; Martin, Bliven y Boisvert, 2008). No se trata de forzar hasta conseguir niveles ideales de funcionamiento (por ejemplo, los tenidos por estadísticamente normales en la población general), sino hasta conseguir el nivel idóneo de funcionamiento de cada persona, en ocasiones superior al que presentaba en el momento en que desarrolló su conducta adictiva. Los adictos, en su gran mayoría, conservan intactas buena parte de sus capacidades cerebrales. Sin embargo, algunas funciones han sufrido alteraciones (por el estilo de vida, por el estrés asociado, por el empobrecimiento estimular, por los efectos farmacológicos de la droga). Nuestro trabajo no consiste tanto en dotarles de algo nuevo como en ayudarles a organizar sus capacidades y a gestionar sus recursos en la vida cotidiana. El protagonista del tratamiento es el propio paciente, pero el responsable del proceso rehabilitador es el terapeuta.

Disponer de un marco conceptual propio permite asimilar conceptos generados en otros ámbitos científicos, pero que se revelan adecuados para la aplicación en la clínica ocupacional. Conceptos como el de empoderamiento (generado en la investigación pedagógica) o enriquecimiento ambiental (generado en la investigación neurocientífica) adquieren un sentido crucial en términos ocupacionales. La rehabilitación cognitiva funcional, generada en la clínica ocupacional en daño cerebral, representa un modelo de intervención adaptable a la clínica de las adicciones. En definitiva, contar con un marco teórico y conceptual propio permite superar el mero nivel observacional-descriptivo, para acceder al plano de la formulación de hipótesis, la explicación de los fenómenos, la predicción en términos científicos y la contrastación/refutación de hipótesis previas.

La Terapia Ocupacional está ya, también en el ámbito de la adicción, en condiciones de dar un paso más allá y reivindicarse como disciplina irrenunciable en la prevención y el tratamiento, desechando definitivamente el plano secundario al que ha sido relegada en tiempos pasados.

\section{ReFERENCIAS BibLIOGRÁFICAS}

Aharonovich, E., Hasin, D. S., Brooks, A. C., Liu, X., Bisaga, A. y Nunes, E. V. (2006). Cognitive deficits predict low treat- ment retention in cocaine dependent patients. Drug \& Alcohol Dependence, 81(3), 313-322. doi: 10.1016/j.drugalcdep.2005.08.003

Alexander, B. K., Coambs, R. B. y Hadaway, P. F. (1978). The effect of housing and gender on morphine self-administration in rats. Psychopharmacology, 58:175-179. Doi: 10.1007/ BF00426903

Allen, C. K., Austin, S. L., David, S. K., Earhart, C. A., McCraith, D. B. y Riska-Williams, L. (2007). Manual for the Allen Cognitive Level Screen-5 (ACLS-5) and Large Allen Cognitive Level Screen (LACLS-5). Camarillo: CA-ACLS and LACLS Committee.

American Occupational Therapy Association. (2011). Definition of occupational therapy practice for the AOTA Model Practice Act. Recuperado de https://www.aota.org/-/media/Corporate/Files/Advocacy/State/Resources/PracticeAct/Model Definition of OT Practice Adopted 41411.pdf

APETO (s. f.). ¿Qué es la Terapia Ocupacional? Recuperado de: http://www.apeto.com/ index.php?option=com_content\&v iew $=$ article\&id $=66 \&$ ltemid $=79$

Arnsten, A. F. (2009). Stress signalling pathways that impair prefrontal cortex structure and function. Nature Reviews $\mathrm{Neu}$ roscience, 10, 410-422. doi:10.1038/nrn2648

Black, R. M. y Wells, S. A. (2007). Culture and occupation: a model of empowerment in occupational therapy. Bethesda: AOTA Press.

Bonikowsky, S., Musto, A., Suteu, K. A., MacKenzie, S. y Dennis, D. (2012). Independence: an analysis of a complex and core construct in occupational therapy. British Journal of Occupational Therapy, 75(4), 188-195. doi: 10.4276/030802212X 13336366278176

Carnero-Pardo C. (2014). ¿Es hora de jubilar al Mini-Mental? Neurología, 29(8), 473-481. doi:10.1016/j.nrl.2013.07.003

Chambers, R. A., Taylor, J. R. y Potenza, M. N. (2003). Developmental neurocircuitry of motivation in adolescence: A critical period of addiction vulnerability. American Journal of Psychiatry, 160, 1041-1052. doi: 10.1176/appi.ajp.160.6.1041

de León Rodríguez, D., Cantero Garlito, P. A. y Zabala Baños, M. (2013). Desempeño ocupacional y calidad de vida en personas con adicción a sustancias. TOG (A Coruña) [revista en Internet], 10(18), [21 p.]. Recuperado de http://www. revistatog.com/num18/pdfs/original1.pdf.

Ersche, K. D., Williams, G.B., Robbins, T. W. yy Bullmore, E. T. (2013). Meta-analysis of structural brain abnormalities associated with stimulant drug dependence and neuroimaging of addiction vulnerability and resilience. Current Opinion in Neurobiology, 23(4), 615-624. doi: 10.1016/j. conb.2013.02.017

Everitt, B. J. y Robbins, T. W. (2005). Neural systems of reinforcement for drug addiction: from actions to habits to compul- 
sion. Nature Neuroscience, 8(11), 1481-1489. doi:10.1038/ nn1579

Fisher, A. (1995). The assessment of motor and process skills (AMPS). Fort Collins, CO: Three Star Press.

Goldstein, R. Z. y Volkow, N. D. (2002). Drug addiction and its underlying neurobiological basis: neuroimaging evidence for the involvement of the frontal cortex. American Journal of Psychiatry, 159, 1642-1652. doi: 10.1176/appi. ajp.159.10.1642

Goldstein, R. Z. y Volkow, N. D. (2011). Dysfunction of the prefrontal cortex in addiction: neuroimaging findings and clinical implications. Nature Reviews Neuroscience, 12, 652669. doi:10.1038/nrn3119

Gutman, S. A. (2006). Why addiction has a chronic, relapsing course. The neurobiology of addiction: Implications for occupational therapy practice. Occupational Therapy in Mental Health, 22(2), 1-29. doi: 10.1300/J004v22n02_01

Hall, W., Carter, A. y Forlini, C. (2015). The brain disease model of addiction: is it supported by the evidence and has it delivered on its promises? Lancet Psychiatry, 2(1):105-110. doi:10.1016/S2215-0366(14)00126-6

HEGOA. (2006). Diccionario de Ayuda Humanitaria y Cooperación Internacional. Instituto sobre desarrollo y cooperación internacional. HEGOA. Recuperado de http://www.dicc. hegoa.ehu.es

Helbig, K. y McKay, E. (2003). An exploration of addictive behaviours from an occupational perspective. Journal of Occupational Science, 10, 140-145. doi: 10.1080/14427591.2003.9686521

Herie, M., Godden, T., Shenfeld, J. y Kelly, C. (2007). Addiction: An information guide. Centre for Addiction and Mental Health. Library and Archives Canada Cataloguing in Publication.

Hunter, B. A., Jason, L. A. y Keys, C. B. (2013). American Journal of Community Psychology, 51(1-2), 91-102. doi: 10.1007/ s10464-012-9499-5

Kelley, A. E. y Berridge, K. C. (2002). The neuroscience of natural rewards: relevance to addictive drugs. Journal of Neuroscience, 22(9), 3306-3311.

Kielhofner, G., Mallinson, T., Crawford, C., Nowak, M., Rigby, M., Henry, A. y Walens, D. (1998). The occupational performance history interview (version 2.0) OPHI-II. Chicago, IL: Model of Human Occupation Clearninghouse; 1998.

Kiepek, N. y Magalhães, L. (2011). Addictions and impulse-control disorders as occupation: A selected literature review and synthesis. Journal of Occupational Science, 18(3), 254276. doi: 10.1080/14427591.2011.581628

Leshner, A. I. (1997). Addiction is a brain disease, and it matters. Science, 278(5335), 45-47. doi: 10.1126/science.278.5335.45
Levy, N. (2013). Addiction is not a brain disease (and it matters). Frontiers in Psychiatry, 4, 24. doi: 10.3389/fpsyt.2013.00024

Martin, L. M., Bliven, M. y Boisvert, R. (2008). Occupational performance, self-esteem, and quality of life in substance addictions recovery. OTJR: Occupation, Participation and Health, 28(2), 81-88. doi: 10.3928/15394492-20080301-05

Nasreddine, Z. S., Phillips, N. A., Bédirian, V., Charbonneau, S., Whitehead, V., Collin, I., ... y Chertkow, H. (2005). The Montreal Cognitive Assessment, MoCA: a brief screening tool for mild cognitive impairment. Journal of the American Geriatrics Society, 53(4), 695-699. doi: 10.1111/j.15325415.2005.53221.x

Navarrete Salas, E. (2010). Ciencia de la Ocupación: caminos y perspectivas. TOG (A Coruña), 7(Supl. 6): S132-S143. Revista en Internet. Recuperado de http://www.revistatog.com/ suple/num6/erna.pdf

Newlin, D. B. y Strubler, K. A. (2007). The habitual brain: An "Adapted Habit" Theory of substance use disorders. Substance Use and Misuse, 42, 503-526. doi: 10.1080/10826080601144606

Olivar Arroyo, Á., Ruiz Sánchez de León, J. M. y Pedrero Pérez, E. J. (2014). Prevención y promoción de la salud en la adicción. En Ruiz Sánchez de León J. M. y Pedrero Pérez, E. J. (eds.), Neuropsicología de la adicción (pp. 125-144). Madrid: Panamericana.

Pedrero-Pérez, E. J., Rojo-Mota, G., Ruiz-Sánchez de León, J. M., Llanero-Luque, M. y Puerta-García, C. (2011). Rehabilitación cognitiva en el tratamiento de las adicciones. Revista de Neurología, 52(7), 163-172.

Pedretti, L. W. (1996). Occupational performance: a model for practice in phisical dysfunction. En Pedretti, L. W., Occupational Therapy: Practice skills for physical dysfunction ( $4^{\mathrm{a}}$ ed.; p. 3-12). Saint Louis: Mosby.

Polatajko, H. J., Backman, C., Baptiste, S., Davis, J., Eftekhar, P., Harvey, A. ... y Connor A. A. (2007). Human occupation in context. En EA Townsend y HJ Polatajko (eds.), Enabling occupation II: Advancing an occupational therapy vision for health, well-being, \& justice through occupation (pp. 37-61). Ottawa, ON: CAOT Publications ACE.

Rao, A. K. (2014). Occupational therapy in chronic progressive disorders: enhancing function and modifying disease. American Journal of Occupational Therapy, 68(3), 251-253. doi:10.5014/ajot.2014.012120

Redish, A. D., Jensen, S. y Johnson, A. (2008). A unified framework for addiction: Vulnerabilities in the decision process. Behavioral Brain Science, 3, 415-487. doi: 10.1017/ S0140525X0800472X

Reed, K. L. (2002). Quick reference to Occupational Therapy (2a ed.). Austin: Pro-Ed. 
Rojo-Mota, G. (2008). Terapia Ocupacional en el tratamiento de las adicciones. Trastornos Adictivos, 10(2), 88-97. doi:10.1016/S1575-0973(08)74550-1

Rojo Mota, G. (2013a). Terapia ocupacional en adicciones: revisión de bases de datos internacionales. TOG (A Coruña) [revista en Internet], 10(17), [14 p.]. Recuperado de http:// www.revistatog.com/num17/pdfs/original3.pdf

Rojo Mota, G. (2013b). Terapia Ocupacional en adicciones y psicopatología asociada. TOG (A Coruña) [revista en Internet], 10(Supl 8), S55-S74. Recuperado de www.revistatog.com/ suple/num8/adicciones.pdf

Rojo Mota, G., Iraurgi Castillo, I. y Sánchez Cabeza, Á. (2011). Rehabilitación cognitiva en el tratamiento de las adicciones. En Pedrero-Pérez, E. J. (coord.), Neurociencia y Adicción (p. 277-298). Madrid: Sociedad Española de Toxicomanías SET.

Rojo-Mota, G., Pedrero Pérez, E. J., Huertas Hoyas, E., Merritt, B. y MacKenzie, D. (2015). Allen Cognitive Level Screen for the classification of subjects treated for addiction. Scandinavian Journal of Occupational Therapy, en prensa.

Rojo Mota, G., Pedrero Pérez, E. J., Ruiz Sánchez de León, J. M., Llanero Luque, M. L., Olivar Arroyo, Á. Puerta-García, C. (2009). Terapia Ocupacional en la rehabilitación de la disfunción ejecutiva en adictos a sustancias. Trastornos Adictivos, 11(2), 96-105. doi:10.1016/S1575-0973(09)72057-4

Rojo Mota, G., Pedrero Pérez, E. J., Ruiz Sánchez de León, J. M., Llanero Luque, M. y Puerta García, C. (2011). Evaluación del desempeño ocupacional en la vida cotidiana en adictos. Creación de un instrumento de medida: el ADO. Adicciones, 23(1), 27-35.

Rojo-Mota, G., Pedrero-Pérez, E. J., Ruiz-Sánchez de León, J. M., Llanero-Luque, M. y Puerta-García, C. (2013). Cribado neurocognitivo en adictos a sustancias: la evaluación cognitiva de Montreal. Revista de Neurología, 56(3), 129-136.

Rojo-Mota, G., Pedrero-Pérez, E. J., Ruiz-Sánchez de León, J. M. y Miangolarra Page, J. C. (2014). Assessment of motor and process skills in daily life activities of treated substance addicts. Scandinavian Journal of Occupational Therapy, 21(6), 458-464. doi: 10.3109/11038128.2014.922610

Sackman, B. S., Sackman, M. M. y DeAngelis, G. G. (1978). Heroin addiction as an occupation: Traditional addicts and heroin-addicted polydrug users. Substance Use and Misuse, 13(3), 427-441. doi: 10.3109/10826087809045259

Sánchez Durán, E. (2011). Instrumentos de evaluación cognitiva en terapia ocupacional. TOG (A Coruña) [revista en Internet], 8(13), [16p.]. Recuperado de http://www.revistatog. com/num13/pdfs/original1.pdf.

Satel, S. y Lilienfeld, S. O. (2014). Addiction and the brain-disease fallacy. Frontiers in Psychiatry, 3(4), 141. doi: 10.3389/ fpsyt.2013.00141
Solinas, M., Thiriet, N., Chauvet, C. y Jaber, M. (2010). Prevention and treatment of drug addiction by environmental enrichment. Progress in Neurobiology, 92(4), 572-592. doi:10.1016/j.pneurobio.2010.08.002

Solinas M, Thiriet N, El Rawas R, Lardeux V, Jaber M. Environmental enrichment during early stages of life reduces the behavioral, neurochemical, and molecular effects of cocaine. Neuropsychopharmacology, 34(5), 1102-1111. doi:10.1038/ npp.2008.51

Substance Abuse \& Mental Health Services Administration's SAMHSA. (2004). National Consensus Statement on Mental Health Recovery. Recuperado de http://www.cibhs.org/ sites/main/files/file-attachments/10_fundamental_components_of_recovery.pdf.

Volkow, N.D. y Baler, R. D. (2014). Addiction science: Uncovering neurobiological complexity. Neuropharmacology, 76, 235-249. doi:10.1016/j.neuropharm.2013.05.007

Wilcock, A. A. (1998). An occupational perspective of health. Thorofare NJ: Slack Incorporated.

Wilcock, A. A. (2001). Occupational science: the key to broadening horizons. Britis Journal of Occupational Therapy, 64(8), 412-417. doi: 10.1177/030802260106400808

World Federation of Occupational Therapists. (2012). Glossary. Recuperado de http://www.wfot.org/aboutus/aboutoccupationaltherapy/definitionofoccupationaltherapy.aspx.

World Health Organization. (1998). Promoción de la Salud. Glosario. Madrid: Subdirección General de Epidemiología, Promoción y Educación para la Salud. Ministerio de Sanidad y Consumo.

Yerxa, E. J. (2000). Occupational science: A renaissance of service to humankind through knowledge. Occupational Therapy International, 7(2), 87-98. doi: 10.1002/oti.109

Yerxa, E. J. (2002). Habits in context: A synthesis, with implications for research in occupational science. OTJR: Occupation, Participation and Health, 22(Suppl 1), S104- S110. doi: $10.1177 / 15394492020220 S 125$

Yücel, M., Solowij, N., Respondek, C., Whittle, S., Fornito, A., Pantelis, C. y Lubman, D. I. (2008). Regional brain abnormalities associated with long-term heavy cannabis use. Archives of General Psychiatry, 65(6), 694-701. doi:10.1001/ archpsyc.65.6.694 